\title{
Physical Exercise, Energy Expenditure and Weight Loss: An Assumption not Always Observed in Practice
}

\author{
Vitor Barreto Paravidino, ${ }^{1,2}$ Mauro Felippe Felix Mediano, ${ }^{3}{ }^{\circledR}$ Rosely Sichieri ${ }^{\circledR}$ \\ Department of Epidemiology, Institute of Social Medicine, State University of Rio de Janeiro, ${ }^{\prime}$ Rio de Janeiro, RJ, Brazil. \\ Department of Physical Education and Sports, Naval Academy - Brazilian Navy, ${ }_{1}^{2}$ Rio de Janeiro, RJ, Brazil. \\ Evandro Chagas National Institute of Infectious Disease, Oswaldo Cruz Foundation, ${ }^{3}$ Rio de Janeiro, RJ, Brazil.
}

The increased prevalence of overweight and obesity observed in recent decades has been attributed mainly to behavioral changes, such as excessive food consumption and reduction in physical activity level over time, leading to a positive energy balance. Due to the technological advances in recent years, less physical effort is required to perform daily tasks. Moreover, the lifestyle has become more sedentary, with an increasing amount of time spent using digital gadgets, such as computers, televisions and mobile phones. However, Westerterp \& Speakman ${ }^{1}$ did not find a decrease in physical activity energy expenditure during the last 30 years preceding their study, despite an increase in the prevalence of obesity. ${ }^{1}$ Other studies have also reported that the total energy expenditure of rural populations was similar to that of populations living in developed countries, despite the clear difference in behavioral patterns, particularly in relation to daily physical activities. ${ }^{2-4}$ This study aimed at presenting the "compensatory effect" as a possible explanation for these findings. When there is an increase in physical activity at a given time, behavioral and metabolic changes take place to maintain an energy setpoint, as presented below.

Physical exercise has been recommended as an important component for the prevention and treatment of obesity. For substantial adults' health benefits, at least 150 minutes of moderate-intensity aerobic exercise, or

\section{Keywords}

Energy Metabolism; Exercise; Weight loss Programs; Overweight; Sedentary behavior; Lifestyle.
75 minutes of vigorous-intensity aerobic exercise; in addition, 2-3 times per week of resistance training is recommended. ${ }^{5}$ However, for a clinically significant reduction in body weight, Donnelly et al., ${ }^{6}$ recommended that individuals should gradually increase the amount of physical activity and achieve a weekly volume of moderate physical exercise of over 250 minutes. ${ }^{6}$ The authors also reported a dose-response effect between physical activity and weight loss.

It is believed that the greater amount of physical exercise (duration and intensity), the higher the total energy expenditure, and consequently, greater loss of body weight. However, energy expenditure through exercise does not explain the variation in body weight observed in the long term. Several studies have shown a weight loss below the expected levels, ${ }^{7,8}$ even in controlled studies with a high rate of patient compliance. ${ }^{9,10}$ These results do not support the classic additive relationship between physical exercise and energy expenditure, thereby raising questions about the real impact of this strategy on weight control. ${ }^{8,11}$

Some theories have tried to explain the reason for lower-than-expected weight loss and the difficulty in maintaining it over time with increased physical activity. In 1998, Rowland proposed the "activitystat" theory and defined it as a homeostatic mechanism, wherein a biological control center would be responsible for controlling the physical activity (or energy expenditure), similarly to other biologically controlled variables such as body temperature. ${ }^{12}$ Whenever an imbalance occurs, the regulatory mechanisms are activated to restore a particular setpoint. According to this theory, an increase

Mailing Address: Vitor Barreto Paravidino

Rua São Francisco Xavier, 524. Postal Code: 20550-013. Pavilhão João Lyra Filho, 70 andar / bloco E, sala 7002, Instituto de Medicina Social, Maracanã, Rio de Janeiro, RJ - Brazil

Email: vparavidino@gmail.com 
in the amount of physical activity at one time would be compensated with less physical activity at another time, to reestablish the balance of the system. This compensatory effect on subsequent spontaneous physical activities has been observed in several studies in children, elderly individuals, and young adults, ${ }^{13-15}$ however, other studies do not corroborate these findings. ${ }^{16}$

Aligned with this theory and in contrast to the classic "additive model," Pontzer et al. (2016) proposed the "restricted model" to explain the relationship between energy expenditure and physical activity. ${ }^{17}$ According to this model, an individual's metabolism adapts in response to the increased physical activity, and above a specific "critical point," the increase in physical activity volume does not cause a concomitant increase in energy expenditure. According to the authors, this compensatory effect can be explained by behavioral (longer periods of sitting than standing or less fidgeting throughout the day compared to a period with no exercise) or metabolic changes (decreased resting metabolic rate, increased muscle efficiency for the same activity demand, or even hormonal changes, such as a decrease in estrogen and testosterone production, and a decrease in the activities of the immune system). ${ }^{17}$

This compensatory phenomenon in relation to physical activity is also observed in some species of birds and mammals. The "energy budget" of these animals is also limited, and any increase in energy expenditure to maintain the basal metabolism would leave a less amount of energy available for other functions, such as flying, fighting, or hunting. ${ }^{18}$

The effect of physical exercise in reducing body weight is further impaired due to compensatory responses on the other side of the energy balance. Martin et al. ${ }^{16}$ observed that overweight individuals submitted to high volumes of physical exercise ( $1760 \mathrm{Kcal} /$ week) for a period greater than six months showed increased appetite and caloric intake compared to the moderate exercise group (700 $\mathrm{Kcal} /$ week) and the group without exercise. These results were corroborated by Myers et al., ${ }^{19}$ who also observed increased hunger and food intake after 12 weeks of daily physical training $(5 \times 500 \mathrm{Kcal} /$ week $){ }^{19}$

Although these compensatory responses are frequently reported in clinical and epidemiological studies, there is significant interindividual variability in weight loss.$^{20} \mathrm{In}$ a study conducted by McNeil et al. (2017) to evaluate the compensatory effect of exercise in 530 postmenopausal women, the authors observed that $9 \%$ of women lost more weight than expected after 12 months of moderate exercise $(150,225$, or 300 minutes per week); $20 \%$ compensated between 0 and $50 \%$, indicating weight loss lower than expected; around $44 \%$ compensated between $50 \%$ and $100 \%$, and $27 \%$ of women presented compensations above $100 \%$, gaining weight at the end of the intervention. ${ }^{21}$ The mechanisms by which individuals respond in such a diverse way to the same stimulus are still a matter of debate; moreover, this compensatory effect seems to be asymmetric, with more intense forces resisting weight loss compared to those acting in response to weight gain.

Therefore, a linear relationship between the prescribed energy deficit and the achieved weight loss should not be assumed. The regulation of the energy balance seems to be a complex and dynamic process, wherein a disturbance in one component can trigger changes in one or more components of energy expenditure and/or food intake. The relationship between individual characteristics (age, sex and nutritional status) and the variables of physical exercise (type of exercise, frequency, intensity and duration) with the compensatory mechanisms requires investigation, as well as the physiological mechanisms responsible for these changes.

The discussion needs to go beyond "eat less and exercise more." Rather, the focus should be on understanding the causal factors and mediating mechanisms for the relationship between physical exercise (and/or diet) and weight loss. Identifying factors that resist weight loss maintenance in some individuals, thereby "disrupting" the entire (or partial) effort, is necessary. Based on the compensatory effects discussed here, the expectation of reducing obesity with programs based exclusively on physical activity should be reduced. ${ }^{8}$ However, regardless of the achieved weight loss response, the health benefits associated with regular physical activity are quite significant, as reported in the literature, and the adoption of an active lifestyle with a decrease in sedentary behaviors should be categorically encouraged.

Long-term clinical trials are still necessary for a better understanding of the effect of physical exercise on energy expenditure, food intake, and body weight, and to identify factors (physiological and behavioral) related to the different responses presented by the individuals. The key question is no longer "if" the compensatory effect occurs, but rather "when," "how," and "what" are the individual characteristics that increase the susceptibility to this phenomenon. 


\section{Potential Conflict of Interest}

No potential conflict of interest relevant to this article was reported.

\section{Sources of Funding}

There were no external funding sources for this study.

\section{Study Association}

This study is not associated with any thesis or dissertation work.

\section{References}

1. Westerterp KR, Speakman JR. Physical activity energy expenditure has not declined since the 1980s and matches energy expenditures of wild mammals. Int J Obes. 2008;32(8):1256-63.

2. Pontzer $\mathrm{H}$, Wood BM, Raichlen DA. Hunter-gatherers as models in public health. Obes Rev. 2018 Dec;19:24-35

3. Dugas LR, Harders R, Merrill S, Ebersole K, Shoham DA, Rush EC, et al. Energy expenditure in adults living in developing compared with industrialized countries: A meta-analysis of doubly labeled water studies. Am J Clin Nutr. 2011;93(2):427-41.

4. Ebersole KE, Dugas LR, Durazo-Arvizu RA, Adeyemo AA, Tayo BO, Omotade OO, et al. Energy expenditure and adiposity in Nigerian and African-American women. Obesity. 2008;16(9):2148-54.

5. Garber CE, Blissmer B, Deschenes MR, Franklin BA, Lamonte MJ Lee I-MM, et al. Quantity and quality of exercise for developing and maintaining cardiorespiratory, musculoskeletal, and neuromotor fitness in apparently healthy adults: Guidance for prescribing exercise. Med Sci Sports Exerc.2011;43(7):1334-59.

6. Donnelly JE, Blair SN, Jakicic JM, Manore MM, Rankin JW, Smith BK Appropriate physical activity intervention strategies for weight loss and prevention of weight regain for adults. Med Sci Sports Exerc. 2009;41(2):459-71.

7. Thorogood A, Mottillo S, Shimony A, Filion KB, Joseph L, Genest J, et al. Isolated aerobic exercise and weight loss: A systematic review and meta-analysis of randomized controlled trials. Am J Med [Internet]. 2011;124(8):747-55.

8. Swift DL, Johannsen NM, Lavie CJ, Earnest CP, Church TS. The role of exercise and physical activity in weight loss and maintenance. Prog Cardiovasc Dis. 2014;56(4):441-7.

9. Church TS, Earnest CP, Skinner JS, Blair SN. Effects of different doses of physical activity oncardiorespiratory fitness among sedentary, overweight or obese postmenopausal women with elevated blood pressure: A randomized controlled trial. J Am Med Assoc. 2007;297(19):2081-91.

10. Manthou E, Gill JM, Wright A, Malkova D. Behavioral compensatory adjustments to exercise training in overweight women. Med Sci Sports Exerc. 2010;42(6):1121-8.

\section{Author Contributions}

Conception and design of the research: Paravidino VB, Mediano MFF, Sichieri R. Acquisition of data: Paravidino VB, Mediano MFF, Sichieri R. Analysis and interpretation of the data: Paravidino VB, Mediano MFF, Sichieri R. Writing of the manuscript: Paravidino VB, Mediano MFF, Sichieri R. Critical revision of the manuscript for intellectual content: Paravidino VB, Mediano MFF, Sichieri R.

11. Mediano MFF, Barbosa JS de O, Moura AS, Willett WC, Sichieri R. A randomized clinical trial of home-based exercise combined with a slight caloric restriction on obesity prevention among women. Prev Med (Baltim) 2010;;51(3-4):247-52.

12. Rowland TW. The biological basis of physical activity. Med Sci Sport Exerc. 1998;30(3):392-9.

13. Paravidino VB, Mediano MFF, Hoffman DJ, Sichieri R. Effect of exercise intensity on spontaneous physical activity energy expenditure in overweight boys: A crossover study. PLoS One. 2016 Jan 1;11(1):e0147141

14. Wang X, Nicklas BJ. Acute impact of moderate-intensity and vigorousintensity exercise bouts on daily physical activity energy expenditure in postmenopausal women. J Obes. 2011;2011:34231.

15. Goran MI, Poehlman ET. Endurance training does not enhance total energy expenditure in healthy elderly persons. Am J Physiol - Endocrinol Metab. 1992;263(5Pt1):E950-7.

16. Martin CK, Johnson WD, Myers CA, Apolzan JW, Earnest CP, Thomas $\mathrm{DM}$, et al. Effect of different doses of supervised exercise on food intake, metabolism, and non-exercise physical activity: The E-MECHANIC randomized controlled trial. Am J Clin Nutr. 2019;110(3):583-92.

17. Pontzer H. Energy constraint as a novel mechanism linking exercise and health. Physiology. 2018;33(6):384-93.

18. Pontzer H. Constrained Total Energy Expenditure and the Evolutionary Biology of Energy Balance. Exerc Sport Sci Rev. 2015;43(3):110-6.

19. Myers A, Dalton M, Gibbons C, Finlayson G, Blundell J. Structured, aerobic exercise reduces fat mass and is partially compensated through energy intake but not energy expenditure in women. Physiol Behav2019;199:56-65.

20. King N, Hopkins M, Caudwell P, Stubbs R, Blundell J. Individual variability following 12 weeks of supervised exercise: identification and characterization of compensation for exercise-induced weight loss. Int J Obes (Lond). 2008;32(1):177-84.

21. McNeil J, Brenner DR, Courneya KS, Friedenreich CM. Dose-response effects of aerobic exercise on energy compensation in postmenopausal women: Combined results from two randomized controlled trials. Int J Obes. 2017;41(8):1196-202. 